\title{
Towards a detailed understanding of the mechanisms of hydrogen retention in beryllium
}

\author{
M. Oberkofler ${ }^{1}$, M. Reinelt ${ }^{1}$, A. Allouche ${ }^{2}$, S. Lindig ${ }^{1}$, \\ and Ch. Linsmeier ${ }^{1}$ \\ ${ }^{1}$ Max-Planck-Institut für Plasmaphysik, EURATOM Association, \\ Boltzmannstraße 2, 85748 Garching b. München, Germany \\ 2 Physique des Interactions Ioniques et Moléculaires, CNRS and \\ Université de Provence, Campus Scientifique de Saint Jérôme, \\ service 242, 13397 Marseille Cedex 20, France
}


Towards a detailed understanding of the mechanisms of hydrogen retention in beryllium2

Abstract. In this paper we present new temperature programmed desorption measurements from implanted single and polycrystalline beryllium as well as new computational results on the diffusion of interstitials through an undisturbed beryllium lattice. The threshold fluence for the appearance of a low-temperature desorption region around $460 \mathrm{~K}$ after implantation of $1 \mathrm{keV} \mathrm{D}$ ions into polycrystalline beryllium is determined to $1.2 \times 10^{21} \mathrm{~m}^{-2}$. In the high temperature release region an implantation energy-dependent shift in desorption temperatures is observed after implantation to very low fluences. It is explained by the variation of the mean distance from the surface for atoms implanted at different energies rather than by changes in the activation energy for detrapping. Morphological investigations of the surface of polycrystalline beryllium after several implantation and annealing cycles show a strong dependence of the damage on the surface-orientation of grains. This dependence correlates with the preferred diffusion of interstitials parallel to basal planes of the beryllium lattice as calculated within the formalism of density functional theory.

PACS codes: 61.80.Jh; 61.72.Hh; 68.43.Vx; 68.55.Ln; 82.20.Pm; 66.30.je; 71.15.Mb; 71.15.Nc;28.52.-s 
Towards a detailed understanding of the mechanisms of hydrogen retention in beryllium3

\section{Introduction}

Understanding of the main mechanisms responsible for the retention of hydrogen atoms implanted into beryllium (Be) is a field of ongoing research. In order to gain further insight in the relevant processes at atomic scale, models have to be developed and tested. However, impure sample surfaces, poorly defined defect densities, and other sources of uncertainty often make it difficult to compare experimental data with values from simulations. Our experimental approach in the past years has been to simplify the system under investigation in order to rule out as many experimental uncertainties as possible. To this aim we have performed measurements on single crystalline Be with welldefined surface oxygen coverages as low as 0.3 monolayers as the only remaining impurity [1]. Temperature programmed desorption (TPD) spectra recorded after implantation

of hydrogen into these well-defined specimens show a sequential release of the retained hydrogen in several temperature steps. These release temperatures are governed by activation energies for the various processes occurring during annealing: detrapping, diffusion, retrapping of hydrogen and self interstitials and, finally, recombination and desorption of hydrogen from the surface. By modelling the release based on rate equations we can extract values for such activation energies from our experiments. Activation energies for individual processes can be calculated ab initio within density functional theory (DFT). This allows a direct comparison of experimental findings with atomistic calculations. This comparison of the experimental and ab initio parameters ultimately should lead to a consistent picture for the hydrogen-related processes in Be.

This work summarizes our recent progress in singling out parameters that influence the observed desorption temperatures. Two topics are addressed. First, we discuss the observed appearance of a low-temperature release peak in the TPD spectra after implantation above the corresponding threshold fluence and compare these new results 
Towards a detailed understanding of the mechanisms of hydrogen retention in beryllium4 from experiments on polycrystalline Be with previously published results from $\mathrm{Be}(11 \overline{2} 0)$ single crystals. Second, TPD experiments with implantation energy-dependent release temperatures performed with a single crystalline sample after implantation up to very low fluences $\left(\sim 10^{19} \mathrm{~m}^{-2}\right)$ are presented. They are discussed in relation to TPD results from single and polycrystalline samples implanted to higher fluences.

Furthermore, recent results from DFT calculations on the diffusion of hydrogen interstitials as well as Be self interstitials in Be are presented. The influence of the resulting diffusional anisotropy on the morphology of the sample surface observed experimentally after numerous implantation and annealing cycles is discussed.

\section{Experimental and computational details}

For the shown TPD spectra polycrystalline or single crystalline samples are implanted with mass-separated deuterium ions $\left(\mathrm{D}_{3}^{+}\right.$or $\left.\mathrm{D}_{2}^{+}\right)$at $1 \mathrm{keV}$ and $3 \mathrm{keV}$ per atom, up to fluences ranging from $2.5 \times 10^{19} \mathrm{~m}^{-2}$ to $2.0 \times 10^{21} \mathrm{~m}^{-2}$. The surface orientation of the single crystalline Be is $(11 \overline{2} 0)$ i.e. the crystal is cut orthogonally to the basal planes. Previous to implantation the surfaces of the samples are cleaned by repeated sputtering with argon ions and subsequent annealing. As observed with in situ X-ray photoelectron spectroscopy (XPS), the only remaining impurity during the whole course of the experiments is a constant amount of beryllium oxide. On the polycrystal the ratio of the relevant XPS signal intensities (Be $1 s$ and $\mathrm{O} 1 s$ ) corresponds to sub-monolayer oxygen coverage. Even though the surface oxygen content of the single crystal was higher than in earlier experiments [2], no influence of this oxide layer on the desorption peaks was observed. Detailed description of the experimental procedure is given elsewhere [2]. The shown spectra from single crystalline beryllium are recorded after implantation with an improved beam spot. The implanted fluence is verified to be homogeneous over an 
Towards a detailed understanding of the mechanisms of hydrogen retention in beryllium5 area of $4 \times 4 \mathrm{~mm}^{2}$ with a steep descent to zero fluence outside this area. The desorption flux is calibrated via the charge collected during implantation with the underlying assumption (verified in [1]) that the retention at small fluences amounts to $80 \%$ of the irradiated fluence. Furthermore, the desorption experiments on the single crystal are performed by using a newly commissioned controller (SCHLICHTING HS170) for obtaining the linear temperature ramp. The heating rate is $42 \mathrm{~K} / \mathrm{min}$ in all shown TPD spectra.

Scanning electron microscopy (SEM) and electron back-scatter diffraction (EBSD) measurements are performed in a field emission gun scanning electron microscope (Helios FEI). To obtain the SEM micrographs the secondary electron signal is recorded with an in-lens detector. EBSD patterns are recorded using a Norlys II detector (HKL/Oxford Instruments).

The simulations of TPD spectra are performed with the rate equation-based code TMAP7 [3]. The implantation depth profiles used in the simulations are calculated with the Monte Carlo code SDTrim.SP $[4,5]$. The computational details for the studies within the framework of density functional theory are given in [6]. In these calculations the stationary state structures are optimized using a quasi-Newton generalized algorithm. All energy calculations are carried out using the Quantum-Espresso package [7]. Activation energies are calculated using the Nudged Elastic Band (NEB) algorithm $[8,9,10]$ implemented in the Quantum-Espresso package.

\section{Results}

\subsection{Threshold fluence for the low-temperature release peak}

Figure 1 shows the temperature dependent release of D from a Be polycrystal previously implanted with $1 \mathrm{keV}$ D ions up to different fluences. The desorption peak around $460 \mathrm{~K}$ 
Towards a detailed understanding of the mechanisms of hydrogen retention in beryllium6 features a threshold behaviour with respect to the irradiated fluence. The peak is absent up to a fluence of $1.17 \times 10^{21} \mathrm{~m}^{-2}$ and appears at a fluence of $1.31 \times 10^{21} \mathrm{~m}^{-2}$ and higher. An analogous parameter study was performed by Reinelt et al. [1] on single crystalline Be cut parallel to the $(11 \overline{2} 0)$ plane. There, the broad high temperature release region was ascribed to detrapping of $\mathrm{D}$ from ion-induced defects (point defects or small clusters) and the low temperature region around $470 \mathrm{~K}$ to the release from regions which have become structurally modified due to implantation above a threshold fluence of $1.22 \times 10^{21} \mathrm{~m}^{-2}$. The structural modifications are supposed to take place in regions of the implantation zone which become supersaturated with $\mathrm{D}$ in the course of the irradiation. The same interpretation applies to the spectra recorded from polycrystalline Be shown here.

The very similar desorption temperatures suggest that the release from both the polycrystalline and the Be $(11 \overline{2} 0)$ occurs from traps of the same nature. The threshold fluence for the release in the low temperature region of the spectrum apparently is not influenced by the presence (or absence) of grain boundaries in the poly- and single crystalline samples. This analogous behaviour can be explained by assuming the supersaturation to take place in the volume of crystallites of the polycrystal. In the inner of crystallites conditions equivalent to the ones present in the single crystal are assumed at the onset of irradiation. It has been argued before that when the kinetic energy of the implanted D falls below the displacement threshold, the impurity atom is trapped after very short diffusion on an atomic length scale. The new results support this assumption: If D diffusion to grain boundaries of the polycrystal is inhibited, similar D concentrations are reached in the volume of crystallites of the polycrystal as in the single crystal at similar fluences.

Furthermore, insight into the possible role of grain boundaries as traps for D in Be can be gained from the shown spectra. If grain boundaries act as additional traps 
Towards a detailed understanding of the mechanisms of hydrogen retention in beryllium7 with an activation barrier higher than the one for the ion induced traps, we expect a corresponding additional release peak at a higher temperature. The fact that such an additional release is not observed leads to the conclusion that if $\mathrm{D}$ is trapped at grain boundaries, the release from there happens at lower temperatures than the release from the ion-induced traps. D atoms possibly released from grain boundaries at lower annealing temperatures could be retrapped in ion-induced defects on their way towards the surface. In this case the release from grain boundaries would remain unobserved.

The fraction of $\mathrm{D}$ detrapping from polycrystalline Be in this low temperature region seems to be higher than for the measurements on the single crystal. This shall, however, not be discussed here since the surface area which was irradiated with a fluence above the threshold is not well known in these experiments. A quantitative analysis of the fractions desorbing in each temperature region will be performed in the future, facilitated by the improved well-defined implantation spot.

\subsection{Dependence of release temperatures on implantation energy}

In a previous publication we discussed the shift of the maximum release temperature observed in TPD spectra from polycrystalline Be after implantation with $\mathrm{D}$ at 1,2 and $3 \mathrm{keV}$ per atom up to fluences of $2 \times 10^{21} \mathrm{~m}^{-2}$ [11]. As can be seen in figure 1 , at these fluences the low temperature release region around $460 \mathrm{~K}$ is well established. Furthermore, the high temperature release region features at least two energetically different release peaks. The nature of these is discussed in [11] and a possible explanation for the shift is given there.

In this work in order to address the retention and release mechanisms leading to the broad desorption region at temperatures around $700-800 \mathrm{~K}$, we record the desorption from a single crystal after implantation up to fluences of $7.8 \times 10^{20}$ and $7.5 \times 10^{20} \mathrm{~m}^{-2}$ with 1 and $2 \mathrm{keV}$ energy per atom, respectively (spectra not shown). 
Towards a detailed understanding of the mechanisms of hydrogen retention in beryllium8 The low temperature release peak is absent in these spectra as the fluence is below the threshold for supersaturation. By choosing this fluence we eliminate a possible influence of $\mathrm{D}$ desorbing at lower temperatures on the release at higher temperatures. In these measurements we find a shift in the maximum release temperature of approx. $40 \mathrm{~K}$. This shift is somewhat smaller (although in the same range) as observed in the experiments on polycrystalline beryllium.

In order to further simplify the initial microscopic configuration at the onset of a desorption experiment, as well as to reduce the number of processes involved during annealing, we further reduce the implantation fluence. The intent is to prepare a parameter region for the defect and hydrogen densities in which only the detrapping and retrapping from and into one single kind of trap with a single activation energy for detrapping controls the observed desorption flux. Figure 2 shows TPD spectra recorded from single crystalline $\mathrm{Be}(11 \overline{2} 0)$ after implantation of $\mathrm{D}$ with energies of 1 and $3 \mathrm{keV}$ per atom up to a fluence of $2.7 \times 10^{19} \mathrm{~m}^{-2}$. At these small fluences the TPD spectra feature a single peak at a temperature around $750 \mathrm{~K}$, i.e. in the lower range of the high temperature region. The shift in the desorption temperature between the two spectra is $24 \mathrm{~K}$.

Assuming only one kind of trap to be responsible for the retention at these low fluences, it is possible to simulate these TPD spectra with one single activation energy. To reproduce the spectra in simulations with TMAP7 we use an activation energy for detrapping of $1.9 \mathrm{eV}$. Upon implantation, irradiation induced point defects and D atoms will stabilize each other, forming immobile traps near the position where the $\mathrm{D}$ atoms are stopped [2]. If this trapping mechanism is assumed to be the only one active for implantation up to very low fluences then the initial trap depth profile corresponds to the implantation depth profile as calculated with SDTrim.SP. 
Towards a detailed understanding of the mechanisms of hydrogen retention in beryllium 9 The maxima of the implantation profiles are at $26 \mathrm{~nm}$ (peak atomic concentration: 0.0065 ) and at $86 \mathrm{~nm}$ (peak atomic concentration: 0.0035), for implantations with $1 \mathrm{keV}$ and $3 \mathrm{keV}$, respectively. Only isotropic diffusion can be treated in TMAP7. The experimental values for the parameters of the isotropic diffusion coefficient $\mathrm{D}=\mathrm{D}_{0}$ $\exp \left(-\mathrm{E}_{D} / \mathrm{RT}\right)$ were used, i.e. $\mathrm{D}_{0}=6.9 \times 10^{-9} \mathrm{~m}^{2} \mathrm{~s}^{-1}$ for the prefactor and $\mathrm{E}_{D}=0.29 \mathrm{eV}$ for the diffusion activation energy [12]. Using those input parameters, the calculations lead to a shift between the two simulated desorption peaks of $30 \mathrm{~K}$ (figure 2) which is in fairly good agreement with the experiment.

Although the TMAP7 simulations provide some insight into the parameters affecting the observed release temperatures, we are not able to reproduce all features of the measured spectra. A main reason for this seems to be that TMAP7 cannot take into account any evolution of the structure and therefore of the trap mobility, and possibly also the trap nature (and therefore of the activation energy for detrapping) in the course of an annealing experiment. Surface morphology investigations hint at structural changes induced in the material in the course of implantation and annealing. In the next paragraph we present our recent results from such investigations.

\subsection{DFT results and orientation dependent morphology}

The NEB calculated barrier for diffusion of a self-interstitial atom in the undisturbed Be lattice is $0.12 \mathrm{eV}$ in the [100] and [010] direction, i.e. parallel to the basal planes, $0.97 \mathrm{eV}$ along the [001] direction, i.e. orthogonal to the basal planes and $0.3 \mathrm{eV}$ along the [111] direction. These low values, in good agreement with the experimental value of $0.15 \mathrm{eV}$, are due to the small crystal cohesion: the Be-Be bonds are so weak that the crystal can adapt itself to the self-interstitial displacement.

The activation barriers for hydrogen diffusion are 0.41 and $0.74 \mathrm{eV}$ for parallel and orthogonal diffusion (with respect to the basal plane), respectively. A hydrogen atom 
Towards a detailed understanding of the mechanisms of hydrogen retention in beryllium 10 can find its path through the crystal at an energy cost comprised between these two limits. This result also is in good agreement with the reported experimental values of $0.29 \mathrm{eV}$ for deuterium diffusion in high-purity Be [13] or $0.32 \mathrm{eV}$ from laser desorption studies of D implanted Be [14].

These anisotropic results for the various diffusion mechanisms are reflected in the surface morphology of the sample developing during repeated bombardment with deuterium (up to fluences above the threshold fluence for structural modifications) followed by annealing up to temperatures well above half the melting point of Be. The left part of figure 3 shows an SEM image of the implanted region of the polycrystalline sample after several experimental runs. The maximum fluence before each TPD run is around or below $2 \times 10^{20} \mathrm{~m}^{-2}$, while the cumulative fluence (summed over all experimental runs) amounts up to approx. $5 \times 10^{22} \mathrm{~m}^{-2}$. Clearly grains with different damage in form of craters of roughly $1 \mu \mathrm{m}$ in diameter are identified. E.g., the marked region to the upper left (full line) exhibits much less defects than the region to the right (broken line). The fact that the dark features visible in the SEM image are holes, not protrusions, is confirmed by atomic force microscopy measurements (not shown here). Outside of the implantation zone no such craters where observed. In the EBSD image of the same region, shown in the right part of figure 3, the orientation of the grains with respect to the basal plane is colour coded. White areas exhibit a (0001) orientation parallel to the surface, dark red areas an orientation of the (0001) plane perpendicular to the surface. A comparison of the two images leads to the conclusion that the damage induced in each grain strongly depends on the orientation of its surface with respect to the basal plane. This correlation points to a considerable influence of the calculated anisotropy in activation energies for diffusion of interstitials on the structural evolution of the material. Such surface orientation- 
Towards a detailed understanding of the mechanisms of hydrogen retention in beryllium11 dependent morphology changes might go along with surface orientation-dependent trap evolution during implantation and/or annealing. This will be further investigated by comparing these results with measurements on single crystals with different surface orientations (e.g. (0001), (10 $0 \overline{1} 0),(11 \overline{2} 0))$.

\section{Summary}

TPD spectra have been recorded from polycrystalline Be and single crystalline Be with a $(11 \overline{2} 0)$ surface orientation after implantation to various fluences. The threshold fluence for the appearance of the low-temperature release peak is the same for the two different specimens. Also, the influence of implantation energy on the release temperatures is similar. A shift in the desorption maximum from the single crystalline sample after implantation up to very low fluences with varying implantation energy is reproduced in TMAP7 simulations by assuming the initial deuterium and trap depth profile for each implantation energy to be equivalent to the implantation depth profiles as calculated by SDTrim.SP. It is concluded that the shift after implantation with different energies at very small fluences is a consequence of the different mean distances of the deuterium atoms to the surface. New DFT results on anisotropic diffusion of interstitials in bulk Be are presented and allow an interpretation of the differences in surface morphology evolution during implantation and release cycles on differently oriented grains. 
Towards a detailed understanding of the mechanisms of hydrogen retention in beryllium 12

\section{References}

[1] M. Reinelt and Ch Linsmeier. Temperature programmed desorption of $1 \mathrm{kev}$ deuterium implanted into clean beryllium. Physica Scripta, T128:111-114, 2007.

[2] M. Reinelt, A. Allouche, M. Oberkofler, and Ch. Linsmeier. Retention mechanisms and binding states of deuterium implanted into beryllium. New Journal of Physics, 11(4):043023, 2009.

[3] J.A. Ambrosek and G.R. Longhurst. Verification and validation of tmap7. Technical Report INEEL/EXT-04-01657, Idaho Falls, September 2004.

[4] W. Eckstein. Computer Simulation of Ion-Solid Interactions, volume 10. Springer Series in Materials Science, 1991.

[5] A. Mutzke W. Eckstein, R. Dohmen and R. Schneider. Sdtrimsp: A monte carlo code for calculating collision phenomena in randomized targets. Report IPP 12/3, 2007.

[6] A. Allouche and Ch. Linsmeier. Quantum study of tungsten interaction with beryllium (0001). Journal of Physics: Conference Series, 117:012002-, 2008.

[7] website http://www.quantum-espresso.org.

[8] Weinan E, Weiqing Ren, and Eric Vanden-Eijnden. String method for the study of rare events. Phys. Rev. B, 66(5):052301, August 2002.

[9] Y. Kanai, A. Tilocca, A. Selloni, and R. Car. First-principles string molecular dynamics: An efficient approach for finding chemical reaction pathways. J. Chem. Phys., 121(8):3359-3367, August 2004.

[10] G. Henkelman and H. Jonsson. A dimer method for finding saddle points on high dimensional potential surfaces using only first derivatives. J. Chem. Phys., 111(15):7010-7022, October 1999.

[11] M. Oberkofler, M. Reinelt, S. Lindig, and Ch. Linsmeier. Structure-dependent deuterium release from ion implanted beryllium: Comparison between be(1 $11-2$-2 $)$ and be(poly). Nuclear Instruments and Methods in Physics Research Section B: Beam Interactions with Materials and Atoms, 267(4):718-722, February 2009.

[12] E. Abramov, M. P. Riehm, D. A. Thompson, and W. W. Smeltzer. Deuterium permeation and diffusion in high-purity beryllium. J. Nucl. Mater., 175(1-2):90-95, December 1990.

[13] A. Kh. Klepikov, I. L. Tazhibaeva, O. G. Romanenko, Y. V. Chikhray, V. P. Shestakov, and E. A. Kenzhin. Influence of loading method on hydrogen retention and release from beryllium. Journal of Nuclear Materials, 258-263(Part 1):798-802, October 1998.

[14] D. Kéroack and B. Terreault. Laser desorption study of deuterium implanted in beryllium. Journal 
Towards a detailed understanding of the mechanisms of hydrogen retention in beryllium 13 of Nuclear Materials, 212-215(Part 2):1443-1447, September 1994. 
Towards a detailed understanding of the mechanisms of hydrogen retention in beryllium 14

\section{Figure captions}

Figure 1

Signal at $4 \mathrm{amu} / \mathrm{q}$ as a function of the sample surface temperature, recorded during annealing of a polycrystalline Be sample previously implanted at room temperature with $1 \mathrm{keV}$ deuterium ions up to various fluences. The linear heating rate is $42 \mathrm{~K} / \mathrm{min}$.

Figure 2

Calibrated deuterium desorption spectra after implantation at room temperature in

single crystalline Be $(11 \overline{2} 0)$ sample up to a fluence of $2.7 \times 19 \mathrm{~m}^{-2}$ at two different ion energies. The smooth curves are the respective simulations with TMAP7.

Figure 3

SEM (left) and EBSD (right) images of the surface in the implantation zone of polycrystalline Be after numerous implantation/desorption cycles. In the EBDS image, white pixels correspond to a (0001)-oriented surface. The angle of deviation from this orientation is colour-coded in red. Not indexed pixels are in black. Two grains are highlighted in each image for comparison: The grain in the upper-left part is oriented orthogonal to the (0 0001$)$ plane (full line), the grain to the right has $(0001)$ orientation (broken line). 
Towards a detailed understanding of the mechanisms of hydrogen retention in beryllium15

Figure 1.

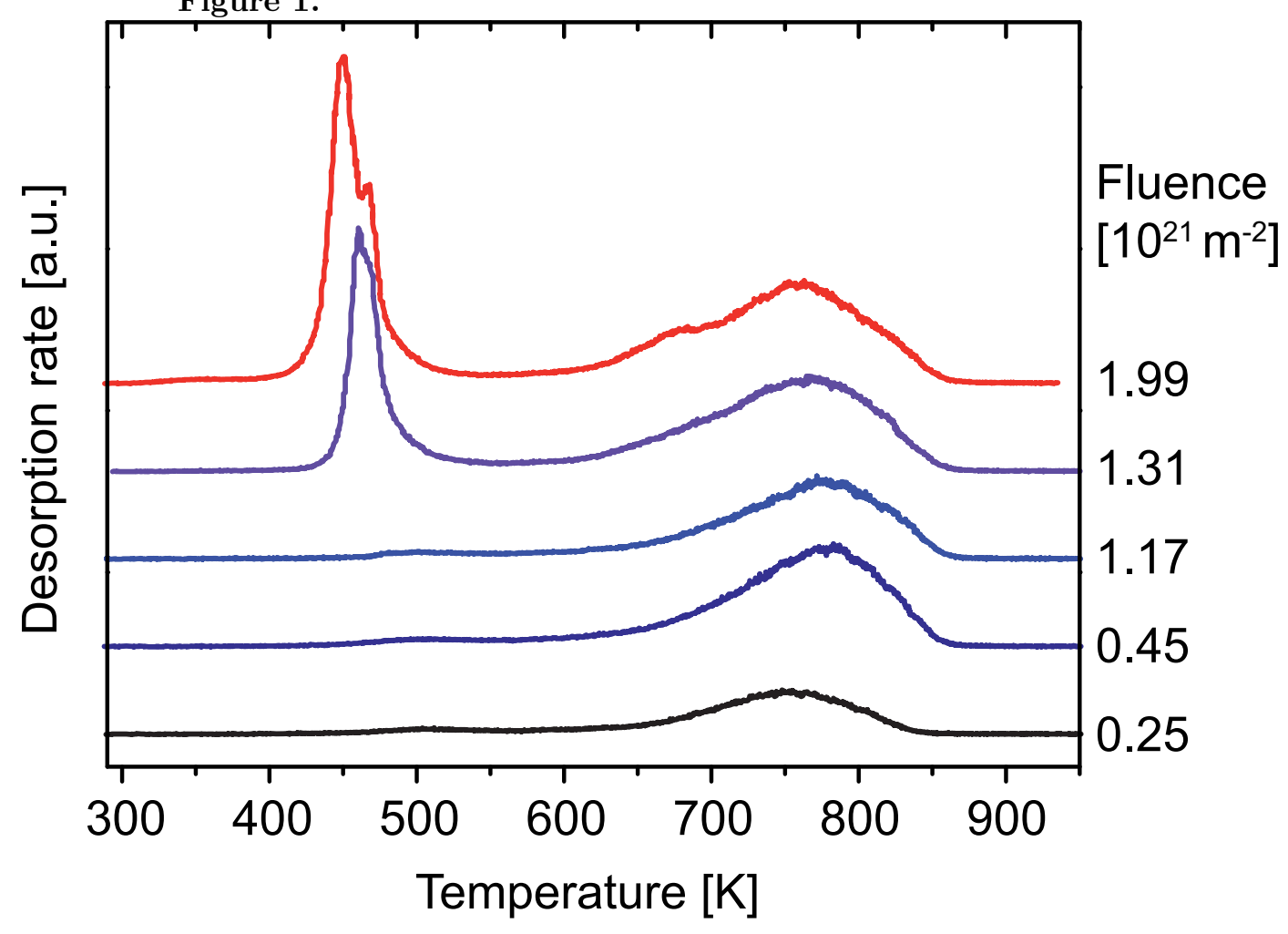

Figure 2.

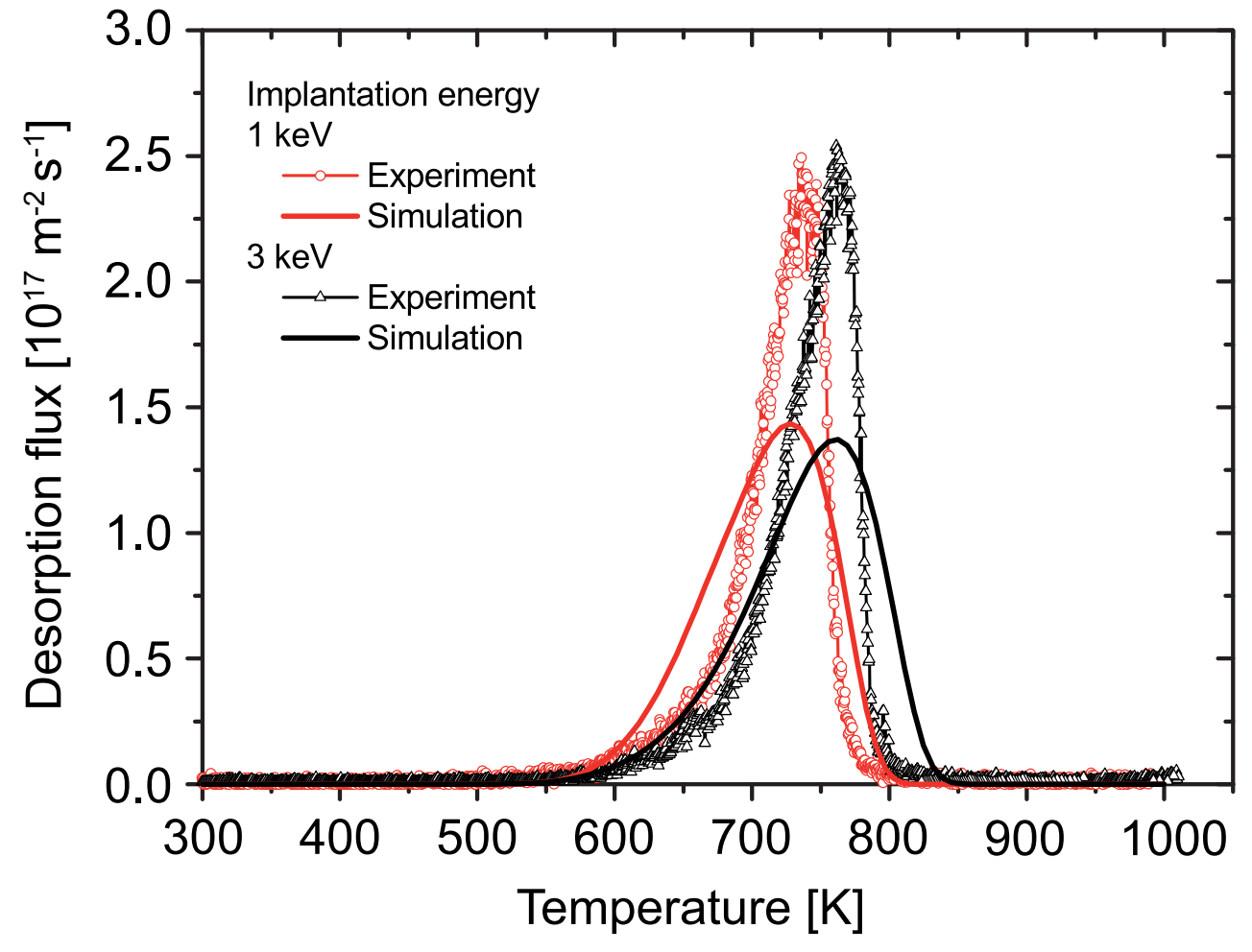


Towards a detailed understanding of the mechanisms of hydrogen retention in beryllium16

Figure 3.
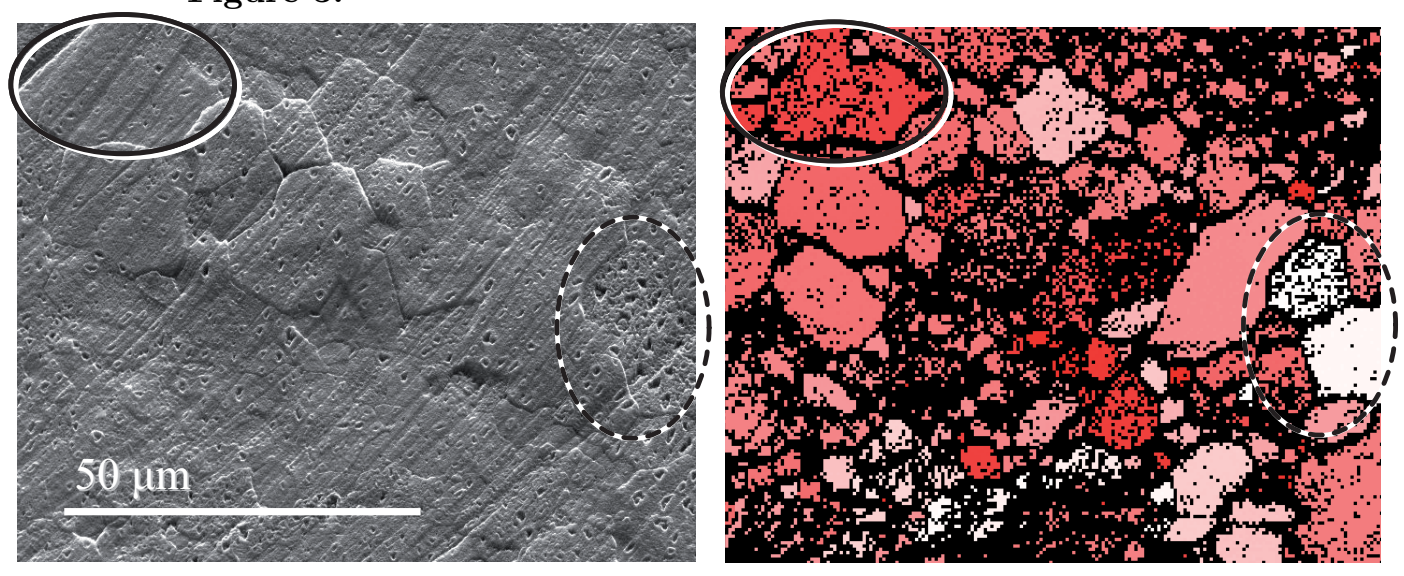